\title{
Intenciones reproductivas y factores asociados con los nacimientos no planeados, Paraguay, 1995-2008
}

\author{
María Mercedes Melian ${ }^{1}$
}

Forma de citar

Melian MM. Intenciones reproductivas y factores asociados con los nacimientos no planeados, Paraguay, 1995-2008. Rev Panam Salud Publica. 2013;33(4):244-51.

RESUMEN Objetivo. Describir las intenciones reproductivas de las mujeres paraguayas entre 1995 y 2008 y determinar las características asociadas con los embarazos no deseados e inoportunos. Métodos. Estudio descriptivo exploratorio basado en datos secundarios de las encuestas nacionales de demografía y salud reproductiva de 1995, 2004 y 2008. Se analizaron los cambios en las características sociodemográficas de las mujeres, el uso de métodos anticonceptivos y la fecundidad, así como de la proporción de nacimientos deseados, inoportunos y no deseados. Mediante dos modelos de regresión logística multifactorial se determinaron las características asociadas con los embarazos no deseados o inoportunos.

Resultados. En 2008, la tercera parte $(33,3 \%)$ de los nacimientos no fueron planeados (25,6\% inoportunos y 7,7\% no deseados). La edad estuvo asociada de forma directa y significativa al deseo de postergar el último nacimiento; el nivel de escolaridad se asoció inversamente con la posibilidad de tener nacimientos inoportunos; $y$ residir en áreas urbanas, con los nacimientos no deseados. El no estar casada o unida a una pareja estable estuvo asociado con considerar el último nacimiento como no deseado o inoportuno, tanto en las mujeres solteras como en las separadas, divorciadas o viudas.

Conclusiones. A pesar del mejoramiento del nivel de escolaridad de las mujeres, su mayor incorporación al trabajo remunerado, el aumento del uso de métodos anticonceptivos y la disminución de la fecundidad, todavía existen grupos de mujeres que no pueden satisfacer sus ideales reproductivos y están expuestas a embarazos no deseados o inoportunos.

Palabras clave Fertilidad; planificación familiar; embarazo no planeado; embarazo no deseado; Paraguay.

De los 210 millones de embarazos que ocurrieron en 1995 en todo el mundo, $38 \%$ fueron no deseados y $22 \%$ terminaron en aborto. Esto representa una pequeña variación con respecto a la situación que imperaba en 2008, cuando se informaron 208 millones de embarazos $\mathrm{y}$, de ellos, $41 \%$ fueron no deseados y $20 \%$ acabaron en aborto. Con respecto

\footnotetext{
1 Centro Paraguayo de Estudios de Población, Departamento de Investigación y Población, Asunción, Paraguay. La correspondencia se debe dirigir a María M. Melian. Correo electrónico: mmelian@cepep.org.py
}

a América Latina y el Caribe, en 2008 se registraron más de 17 millones de embarazos, de los cuales alrededor de 58\% (cerca de 10 millones) no eran deseados y $22 \%$ (alrededor de 4 millones) finalizaron en abortos electivos (1). En esta subregión, 91\% de las adolescentes de 15-19 años no casadas y activas sexualmente necesitaban anticonceptivos $y$, de ese total, $40 \%$ tenía necesidades insatisfechas de planificación familiar (2).

En Paraguay, donde el aborto está penalizado por el Código Penal -excepto, cuando se trata de salvar la vida de la mujer puesta en peligro por el embarazo o el parto-, la planificación familiar está reconocida como un derecho constitucional y el país cuenta con un plan nacional quinquenal de salud sexual y reproductiva (3-5).

Reducir el número de los nacimientos no planeados - ya sean inoportunos (no deseados en ese momento, pero que se hubieran querido tener más adelante) o no deseados (no se querían en ningún momento) - justifica en gran parte los esfuerzos realizados por los gobiernos y las agencias internacionales para promo- 
ver el acceso universal a los anticonceptivos (6). El número de hijos deseados es uno de los factores que influyen en la cantidad de embarazos no deseados en una población. En sociedades que se inclinan por tener familias numerosas, la tasa de embarazos no deseados tiende a ser más baja que en otras donde se prefiere un menor número de hijos (7).

Los valores de fecundidad deseada permiten estimar la medida en que variaría la fecundidad de una población si las mujeres concretasen sus preferencias reproductivas (8). Los estudios sobre las intenciones y las preferencias reproductivas de las mujeres se han basado principalmente en metodologías diseñadas para captar de manera más precisa la fecundidad deseada $(8,9)$ y la no deseada $(10,11)$ a partir de datos de encuestas nacionales. Para ello se han empleado datos de las encuestas internacionales de fecundidad, las encuestas nacionales de demografía y salud de la década de 1970, y las encuestas nacionales de salud reproductiva de inicios de la década de 1980. La inclusión en ellas de preguntas dirigidas a conocer las preferencias sobre el tamaño ideal de la familia, el deseo de tener más hijos o la intención respecto a los embarazos en el momento en que estos ocurrieron posibilitan estimar directa o indirectamente las tasas de fecundidad deseada y no deseada, y los porcentajes de nacimientos deseados, inoportunos y no deseados (12).

En los Estados Unidos de América se emplearon encuestas específicas de fecundidad para conocer los embarazos no deseados y no planeados (inoportunos o no deseados) para explicar las tendencias y las diferencias encontradas en las tasas de fecundidad (13). Si bien en 1941, 1955 y 1960 se midió solamente la fecundidad no deseada en mujeres casadas, en la Encuesta Nacional de Fecundidad de 1965 se incluyó el concepto de nacimientos inoportunos y en la Encuesta Nacional de Crecimiento Familiar de 1973, 1976, 1982 y 1988 se preguntó a todas las mujeres acerca de los nacimientos no deseados o inoportunos. En 1995, esa encuesta mostró que aproximadamente la tercera parte de los nacidos vivos en los Estados Unidos era resultado de embarazos no deseados o inoportunos (14).

A partir de los datos de una encuesta internacional sobre fecundidad se pudo constatar que, de haberse evitado todos los nacimientos no deseados, los nive- les de fecundidad habrían disminuido entre $25 \%$ y $33 \%$ en cinco de los seis países de Asia y América Latina analizados (10).

Un análisis de la fecundidad no deseada a partir de los datos de 59 encuestas -entre ellas encuestas internacionales sobre fecundidad, encuestas nacionales de demografía y salud y encuestas nacionales de salud reproductiva-, realizadas entre 1970 y 2000 (15), mostró que la fecundidad no deseada era siempre más alta en América Latina que en otras regiones. En ese estudio, Paraguay presentó la menor proporción de nacimientos no deseados (21\%), en contraste con Bolivia (61\%), Colombia (40\%), Haití $(40 \%)$ y Perú $(40 \%)$.

En otro estudio sobre Paraguay, realizado a partir de los datos de la Encuesta Mundial de Fecundidad de 1979 (16), se encontró que solo $5 \%$ de las mujeres que no querían tener más hijos utilizaba algún método anticonceptivo, lo que podría indicar que más mujeres paraguayas querían tener familias numerosas que otras mujeres de América Latina y tendrían una menor fecundidad no deseada. No obstante, se debe señalar que el porcentaje de embarazos antes del tiempo deseado fue mayor en los segundos embarazos respecto a los primeros, debido fundamentalmente a que los primeros generalmente fueron planeados (17).

El objetivo del presente trabajo es describir las intenciones reproductivas de las mujeres paraguayas entre 1995 y 2008 y determinar las características asociadas con los embarazos no deseados e inoportunos.

\section{MATERIALES Y MÉTODOS}

En este estudio descriptivo exploratorio se emplearon datos de las encuestas nacionales de demografía y salud reproductiva realizadas por el Centro Paraguayo de Estudios de Población en los años 1995, 2004 y 2008 (18-20), como parte del programa de encuestas nacionales de salud reproductiva que se llevó a cabo con la asistencia técnica del Centro para la Prevención y el Control de Enfermedades (CDC) de los Estados Unidos.

En esas tres encuestas se empleó un diseño de muestreo probabilístico en varias etapas, con selección aleatoria de los conglomerados, los hogares y las mujeres en edad fértil, de 15 a 44 años, y se basaron en muestras de 5 966, 7321 y 6540 mujeres, respectivamente, representativas de todo el país.

Para determinar la intención reproductiva de las mujeres, en el cuestionario se incluyó la pregunta: “Cuando quedó embarazada, ¿quería usted tener ese hijo entonces, quería esperar más tiempo o no quería más hijos?" Se clasificó el embarazo (nacimiento) como deseado si la mujer respondió que deseaba quedar embarazada en ese momento, inoportuno si respondió que quería esperar más tiempo antes de embarazarse o no deseado si contestó que no quería tener más hijos. Los casos analizados se limitaron a los nacimientos ocurridos en los 36 meses previos a las encuestas (2 859 en 1995, 2430 en 2004 y 1812 en 2008).

\section{Análisis}

El análisis se desarrolló en tres etapas: primero, se compararon los resultados para detectar posibles cambios en las características sociodemográficas de las mujeres (nivel educacional, estado conyugal, situación laboral y número de hijos vivos), el uso de métodos anticonceptivos según la edad y la situación laboral, y el nivel de fecundidad ${ }^{2}$ según el área de residencia de las mujeres entrevistadas. En una segunda etapa, se realizó el análisis descriptivo de la proporción de nacimientos deseados, inoportunos y no deseados. Por último, se determinaron las características asociadas con las mujeres que tuvieron embarazos no deseados, mediante regresión logística multifactorial con los datos del último nacimiento declarado en la encuesta de 2008, a fin de reducir los posibles errores de declaración (22). Se calculó la razón de posibilidades (odds ratio, OR) con sus intervalos de confianza de 95\% (IC95\%).

Se construyeron dos modelos de regresión logística multifactorial. En el primero, la muestra se limitó a las mujeres con nacimientos deseados (grupo de referencia) y no deseados (variable dependiente). En el segundo modelo, la muestra incluyó a las mujeres con nacimientos deseados (grupo de referencia) e inoportunos (variable dependiente). En estos modelos se utilizaron las variables independientes que mostraron asociación significativa con la variable dependiente correspondiente, según la prueba de la

\footnotetext{
2 Para medir la fecundidad se utilizó la tasa global de fecundidad (21).
} 
ji al cuadrado. Se incluyó también la variable área de residencia debido a la asociación mostrada en análisis bifactoriales anteriores (20) y por ser un factor de predicción de embarazos no deseados (23).

Las variables independientes analizadas fueron: edad, área de residencia, vel socioeconómico, situación laboral y número de hijos vivos. La edad se clasificó en tres grupos (15-19, 20-29 y 30-44 años). El área de residencia se clasificó en urbana o rural; el estado conyugal, en soltera, casada/unida o separada/ divorciada/viuda; el nivel educacional se clasificó en cuatro grupos principales: menos de 6 años de estudio (educación básica inconclusa), 6 años terminados (educación básica vencida), de 7 a 11 años (educación secundaria inconclusa) y 12 años de estudio o más; y el nivel socioeconómico, en bajo, medio y alto, según los bienes duraderos que poseía y los servicios de las viviendas (24-26).

Para la variable situación laboral se consideró que una mujer trabajaba si recibía remuneración (independientemente de si realizaba su labor dentro o fuera de su casa). Para la variable hijos actualmente vivos se tomó el número de hijos vivos $(1,2, \ldots, n)$. estado conyugal, nivel educacional, ni-

Todos los resultados se ponderaron para compensar los errores que podía introducir el tipo de muestreo, mediante un factor de ponderación inversamente proporcional a la probabilidad de selección de una vivienda y, dentro de ella, de una mujer en particular.

\section{RESULTADOS}

\section{Cambios en la condición socioeconómica de las mujeres}

Entre 1995 y 2008, la proporción de mujeres sin educación o que solo completaron dos años de estudio se redujo en más de $60 \%$ (de $7,7 \%$ a 2,7\%), mientras el grupo con tres a cinco años de estudio disminuyó de $24,8 \%$ a $10,3 \%$ (cuadro 1 ). Se duplicó el porcentaje de mujeres que completó la secundaria o cursó algún año de universidad (de $16,9 \%$ a $39,1 \%$ ) y la proporción que realizaba alguna actividad laboral remunerada fuera de la casa aumentó en más de 50\% (de 25,6\% a $39,3 \%$ ). En relación con el estado conyugal, el porcentaje de mujeres casadas o en unión consensual se redujo de $60,8 \%$ a $53,9 \%$ y el de solteras se incrementó de $32,1 \%$ a $38,7 \%$. Se observó un incremento de más de $20 \%$ en la proporción de mu-

CUADRO 1. Características de las mujeres de 15 a 44 años de edad, según las encuestas analizadas, Paraguay, 1995, 2004 y 2008

\begin{tabular}{lccc}
\hline \multicolumn{1}{c}{ Característica } & $\begin{array}{c}\text { ENDSR } 1995,{ }^{\mathrm{a}} \\
\%\end{array}$ & $\begin{array}{c}\text { ENDSSR } 2004,{ }^{\mathrm{b}} \\
\%\end{array}$ & $\begin{array}{c}\text { ENDSSR 2008, } \\
\%\end{array}$ \\
\hline Años de estudio terminados & & & \\
$0-2$ & 7,7 & 3,9 & 2,7 \\
$3-5$ & 24,8 & 12,6 & 10,3 \\
6 & 27,5 & 21,7 & 18,1 \\
$7-11$ & 23,1 & 29,3 & 29,8 \\
$\geq 12$ & 16,9 & 32,5 & 39,1 \\
Estado conyugal & & & 53,9 \\
Casada/unida & 60,8 & 53,7 & 7,5 \\
Separada/divorciada/viuda & 7,0 & 7,5 & 38,7 \\
Soltera & 32,1 & 38,9 & 52,1 \\
Situación laboral & & & 39,3 \\
Sin trabajo remunerado & 56,7 & 54,9 & 8,6 \\
Trabaja fuera de la casa & 25,6 & 31,6 & \\
Trabaja dentro de la casa & 17,7 & 13,5 & 41,6 \\
Hijos actualmente vivos & & & 18,8 \\
0 & 33,8 & 40,2 & 15,4 \\
1 & 15,6 & 16,6 & 10,8 \\
2 & 14,5 & 14,7 & 6,0 \\
3 & 11,7 & 10,6 & 7,4 \\
4 & 8,2 & 7,2 & \\
$\geq 5$ & 16,2 & 10,8 &
\end{tabular}

a ENDSR 1995: Encuesta Nacional de Salud Reproductiva, 1995 ( $n=5$ 966).

b ENDSSR 2004: Encuesta Nacional de Demografía y Salud Sexual y Reproductiva, 2004 ( $n=7321$ ).

c ENDSSR 2008: Encuesta Nacional de Demografía y Salud Sexual y Reproductiva, 2008 ( $n=6540)$. jeres que postergaron el nacimiento del primer hijo (de $33,8 \%$ a $41,6 \%$ ) (cuadro 1 ).

\section{Aumento en el uso de anticonceptivos}

El aumento en el uso de anticonceptivos en 2008 con respecto a 1995, en general, fue de cerca de $50 \%$ (de $53,5 \%$ a $79,4 \%$ ) y de más de $60 \%$ (de $43,8 \%$ a $70,7 \%$ ) con los métodos denominados modernos, como las píldoras, los inyectables, los dispositivos intrauterinos, el condón, la ligadura de trompas y los espermicidas (cuadro 2).

Esta práctica se extendió a todos los sectores sociales; no obstante, el cambio porcentual registrado fue mayor (38 puntos porcentuales) en las mujeres del nivel socioeconómico bajo, quienes en 1995 tenían un menor porcentaje de uso $(31,4 \%)$ y llegaron a $69,3 \%$ en 2008 (cuadro 2).

\section{Descenso de la fecundidad}

La tasa global de fecundidad en Paraguay disminuyó significativamente $(P<0,05)$ de 4,2 hijos por mujer en 1995 a 2,5 hijos en 2008 (cuadro 3). Esta reducción se observó en todos los grupos, independientemente de las características sociodemográficas de las mujeres; sin embargo, el cambio no fue homogéneo. Las mujeres que vivían en áreas urbanas, con más educación, de nivel socioeconómico alto y que trabajaban fuera de la casa ya tenían menor fecundidad en 1995 y continuaron reduciendo su descendencia, mientras que en las mujeres de áreas rurales, con menor educación, nivel económico medio o bajo, y sin trabajo remunerado el porcentaje de cambio fue mayor.

Se estrechó la brecha entre los extremos más alto y más bajo de la tasa global de fecundidad. En 1995, la mayor diferencia en cuanto a la fecundidad se observó en las mujeres de menor educación (6,3 hijos en promedio) y las de mayor educación (2,2 hijos); esta diferencia de 4,1 hijos en promedio se redujo a 1,5 en 2008 como resultado de la mayor disminución del número de hijos en las mujeres con menor nivel educacional con respecto a las mujeres de mayor nivel (cuadro 3).

\section{Nacimientos deseados, inoportunos y no deseados}

En las tres encuestas analizadas se encontró que más de dos tercios de 
CUADRO 2. Frecuencia del uso de métodos anticonceptivos, según su tipo y el nivel socioeconómico, en mujeres casadas/unidas de 15 a 44 años de edad, Paraguay, 1995, 2004 y 2008

\begin{tabular}{|c|c|c|c|c|c|c|c|c|c|}
\hline \multirow[b]{2}{*}{ Característica } & \multicolumn{3}{|c|}{ ENDSR $1995^{a}$} & \multicolumn{3}{|c|}{ ENDSSR $2004^{b}$} & \multicolumn{3}{|c|}{ ENDSSR $2008^{c}$} \\
\hline & $\begin{array}{l}\text { No. de } \\
\text { casos }\end{array}$ & $\begin{array}{c}\text { Uso } \\
\text { actual, }^{\mathrm{d}} \\
\%\end{array}$ & $\begin{array}{c}\text { Uso } \\
\text { moderno, } \\
\%\end{array}$ & $\begin{array}{l}\text { No. de } \\
\text { casos }\end{array}$ & $\begin{array}{c}\text { Uso } \\
\text { actual, } \\
\%\end{array}$ & $\begin{array}{c}\text { Uso } \\
\text { moderno, } \\
\%\end{array}$ & $\begin{array}{l}\text { No. de } \\
\text { casos }\end{array}$ & $\begin{array}{c}\text { Uso } \\
\text { actual, } \\
\%\end{array}$ & $\begin{array}{c}\text { Uso } \\
\text { moderno, } \\
\%\end{array}$ \\
\hline Total & 4194 & 53,5 & 43,8 & 4564 & 72,8 & 60,5 & 3948 & 79,4 & 70,7 \\
\hline \multicolumn{10}{|l|}{ Edad (años) } \\
\hline $15-19$ & 246 & 37,2 & 30,5 & 232 & 60,8 & 47,5 & 234 & 70,9 & 65,6 \\
\hline $20-24$ & 703 & 49,2 & 39,9 & 785 & 71,3 & 61,6 & 625 & 81,2 & 74,0 \\
\hline $25-29$ & 964 & 56,1 & 48,1 & 973 & 71,6 & 62,0 & 884 & 82,1 & 75,0 \\
\hline $30-34$ & 988 & 57,4 & 47,3 & 968 & 73,6 & 62,8 & 831 & 82,0 & 72,4 \\
\hline $35-39$ & 751 & 57,7 & 47,7 & 891 & 74,5 & 59,2 & 729 & 80,8 & 70,3 \\
\hline $40-44$ & 542 & 48,0 & 37,1 & 715 & 75,7 & 60,2 & 625 & 72,4 & 62,2 \\
\hline \multicolumn{10}{|l|}{ Nivel socioeconómico ${ }^{f}$} \\
\hline Bajo & 1727 & 40,4 & 31,4 & 1791 & 62,8 & 50,0 & 1654 & 76,8 & 69,3 \\
\hline Medio & 1253 & 53,9 & 46,0 & 1513 & 75,6 & 62,9 & 1325 & 82,3 & 72,5 \\
\hline Alto & 1214 & 67,5 & 55,5 & 1260 & 79,6 & 68,1 & 969 & 79,1 & 70,3 \\
\hline
\end{tabular}

a ENDSR 1995: Encuesta Nacional de Salud Reproductiva, 1995.

b ENDSSR 2004: Encuesta Nacional de Demografía y Salud Sexual y Reproductiva, 2004.

c ENDSSR 2008: Encuesta Nacional de Demografía y Salud Sexual y Reproductiva, 2008.

d Porcentaje de mujeres activas sexualmente casadas o unidas, que usaban anticonceptivos en el momento de la entrevista o en los 30 días previos.

e Píldoras, inyectables, dispositivos intrauterinos, condón, ligadura de trompas y espermicidas.

† Según los bienes duraderos que poseía y los servicios de las viviendas (24-26).

CUADRO 3. Tasa global de fecundidad y porcentaje de cambio, según las características seleccionadas, Paraguay, 1995, 2004 y 2008

\begin{tabular}{|c|c|c|c|c|}
\hline \multirow[b]{2}{*}{ Característica } & \multicolumn{3}{|c|}{ Tasa global de fecundidad ${ }^{a}$} & \multirow{2}{*}{$\begin{array}{c}\text { Variación en } \\
\text { la TGF } \\
2008 / 1995 \\
\%\end{array}$} \\
\hline & $\begin{array}{c}\text { ENDSR } \\
1995^{\mathrm{b}}\end{array}$ & $\begin{array}{c}\text { ENDSSR } \\
2004^{\circ}\end{array}$ & $\begin{array}{c}\text { ENDSSR } \\
2008^{\mathrm{d}}\end{array}$ & \\
\hline Total & 4,2 & 2,9 & 2,5 & $-40,5$ \\
\hline \multicolumn{5}{|l|}{ Área de residencia } \\
\hline Urbana & 3,1 & 2,5 & 2,2 & $-29,0$ \\
\hline Rural & 5,4 & 3,7 & 3,0 & $-44,4$ \\
\hline Diferencia entre el valor más alto y el más bajo & 2,3 & 1,2 & 0,8 & \\
\hline \multicolumn{5}{|l|}{ Años de estudio terminados } \\
\hline $0-2$ & 6,3 & 5,1 & 3,5 & $-44,4$ \\
\hline $3-5$ & 5,3 & 4,0 & 3,6 & $-32,1$ \\
\hline 6 & 4,5 & 3,8 & 3,0 & $-33,3$ \\
\hline $7-11$ & 3,2 & 2,9 & 2,7 & $-15,6$ \\
\hline$\geq 12$ & 2,2 & 2,1 & 2,0 & $-9,1$ \\
\hline Diferencia entre el valor más alto y el más bajo & 4,1 & 3,0 & 1,5 & \\
\hline \multicolumn{5}{|l|}{ Nivel socioeconómico ${ }^{e}$} \\
\hline Bajo & 6,2 & 4,6 & 3,5 & $-43,5$ \\
\hline Medio & 4,0 & 2,6 & 2,2 & $-45,0$ \\
\hline Alto & 2,5 & 2,0 & 1,7 & $-32,0$ \\
\hline Diferencia entre el valor más alto y el más bajo & 3,7 & 2,6 & 1,8 & \\
\hline \multicolumn{5}{|l|}{ Situación laboral } \\
\hline Sin trabajo remunerado & 5,1 & 3,5 & 3,1 & $-39,2$ \\
\hline Trabaja dentro de la casa & 3,6 & 2,5 & 2,3 & $-36,1$ \\
\hline Trabaja fuera de la casa & 2,6 & 2,3 & 1,8 & $-30,8$ \\
\hline Diferencia entre el valor más alto y el más bajo & 2,5 & 1,2 & 1,3 & \\
\hline \multicolumn{5}{|c|}{$\begin{array}{l}\text { a La tasa global de fecundidad, es el número de hijos que en promedio tendría una mujer de una cohorte } \\
\text { res que durante su vida fértil tuvieran sus hijos de acuerdo a las tasas de fecundidad por edad del perí } \\
\text { estuviera expuesta a riesgos de mortalidad desde el nacimiento hasta el término del período fértil (21). } \\
\text { b ENDSR 1995: Encuesta Nacional de Salud Reproductiva, } 1995(n=5966) \text {. } \\
\text { c ENDSSR 2004: Encuesta Nacional de Demografía y Salud Sexual y Reproductiva, } 2004 \text { ( } n=7321) \text {. } \\
\text { d ENDSSR 2008: Encuesta Nacional de Demografía y Salud Sexual y Reproductiva, } 2008 \text { ( } n=6540) \text {. } \\
\text { e Según los bienes duraderos que poseía y los servicios de las viviendas (24-26). }\end{array}$} \\
\hline
\end{tabular}

los nacimientos fueron deseados. Sin embargo, los nacimientos inoportunos, es decir, de madres que hubieran preferido posponer el embarazo para más adelante, aumentaron entre 1995 y 2008 en más de $50 \%$ (de $16,5 \%$ a $25,6 \%$ ), mientras que los no deseados se mantuvieron sin grandes variaciones (cuadro 4). En 2008, 44,9\% de los partos de adolescentes —de 15 a 19 años- se debieron a embarazos no planeados, ya fueran inoportunos $(41,8 \%)$ o no deseados $(3,1 \%)$. De manera similar, más de la mitad $(50,9 \%)$ de los niños nacidos de madres solteras en 2008 fueron nacimientos inoportunos $(43,9 \%)$ o no deseados $(7,0 \%)$ (cuadro 4$)$.

\section{Factores asociados con los nacimientos inoportunos o no deseados}

El estado conyugal y el número de nacimientos previos se asociaron significativamente tanto con los nacimientos inoportunos como con los no deseados. El área de residencia solo se asoció con los nacimientos no deseados, mientras que la edad de las madres y los años de educación se asociaron con los nacimientos inoportunos. El nivel socioeconómico y la situación laboral no presentaron asociación significativa en ninguno de los dos modelos (cuadro 5). 
CUADRO 4. Porcentaje de nacimientos ocurridos en el período de 36 meses anteriores a la encuesta, por intención reproductiva y características de las mujeres, Paraguay, 1995, 2004 y 2008

\begin{tabular}{|c|c|c|c|c|c|c|c|c|c|}
\hline \multirow[b]{2}{*}{ Característica } & \multicolumn{3}{|c|}{ Nacimientos deseados } & \multicolumn{3}{|c|}{ Nacimientos inoportunos } & \multicolumn{3}{|c|}{ Nacimientos no deseados } \\
\hline & $\begin{array}{c}\text { ENDSR } \\
1995{ }^{\mathrm{a}} \\
\%\end{array}$ & $\begin{array}{c}\text { ENDSSR } \\
2004,{ }^{b} \\
\%\end{array}$ & $\begin{array}{c}\text { ENDSSR } \\
2008^{c}{ }^{\circ} \\
\%\end{array}$ & $\begin{array}{c}\text { ENDSR } \\
1995, \\
\%\end{array}$ & $\begin{array}{c}\text { ENDSSR } \\
2004 \\
\%\end{array}$ & $\begin{array}{c}\text { ENDSSR } \\
2008 \\
\%\end{array}$ & $\begin{array}{c}\text { ENDSR } \\
1995, \\
\%\end{array}$ & $\begin{array}{c}\text { ENDSSR } \\
2004 \\
\%\end{array}$ & $\begin{array}{c}\text { ENDSSR } \\
2008 \\
\%\end{array}$ \\
\hline Total & 76,7 & 68,4 & 66,7 & 16,5 & 21,2 & 25,6 & 6,7 & 10,4 & 7,7 \\
\hline \multicolumn{10}{|l|}{ Área de residencia } \\
\hline Urbana & 75,7 & 66,5 & 65,8 & 17,2 & 22,7 & 25,0 & 7,1 & 10,8 & 9,2 \\
\hline Rural & 78,1 & 70,9 & 67,8 & 15,7 & 19,2 & 26,4 & 6,2 & 10,0 & 5,7 \\
\hline \multicolumn{10}{|l|}{ Edad (años) } \\
\hline $15-19$ & 78,8 & 60,6 & 55,2 & 18,2 & 35,5 & 41,8 & 3,0 & 3,9 & 3,1 \\
\hline $20-24$ & 79,7 & 65,1 & 63,6 & 17,0 & 28,1 & 32,9 & 3,3 & 6,8 & 3,5 \\
\hline $25-29$ & 73,9 & 72,8 & 68,3 & 20,8 & 19,8 & 26,9 & 5,3 & 7,5 & 4,8 \\
\hline $30-34$ & 77,2 & 70,5 & 72,1 & 14,5 & 14,0 & 18,6 & 8,3 & 15,5 & 9,3 \\
\hline $35-39$ & 75,7 & 73,4 & 70,8 & 12,0 & 14,0 & 9,4 & 12,2 & 12,6 & 19,8 \\
\hline $40-44$ & 73,7 & 60,2 & 65,7 & 10,3 & 6,0 & 7,2 & 16,0 & 33,8 & 27,1 \\
\hline \multicolumn{10}{|l|}{ Años de estudio terminados } \\
\hline $0-2$ & 70,0 & 63,8 & 72,4 & 18,9 & 17,1 & 12,6 & 11,2 & 19,0 & 14,9 \\
\hline $3-5$ & 75,0 & 69,6 & 67,7 & 16,8 & 16,4 & 24,0 & 8,2 & 14,0 & 8,3 \\
\hline 6 & 76,7 & 67,3 & 66,8 & 17,9 & 21,0 & 23,4 & 5,4 & 11,7 & 9,8 \\
\hline $7-11$ & 80,2 & 64,9 & 65,8 & 14,0 & 25,0 & 26,0 & 5,8 & 10,1 & 8,2 \\
\hline$\geq 12$ & 84,2 & 73,1 & 66,3 & 13,2 & 22,0 & 28,8 & 2,6 & 4,9 & 5,0 \\
\hline \multicolumn{10}{|l|}{ Estado conyugal } \\
\hline Casada/unida & 76,8 & 70,9 & 70,7 & 16,6 & 18,9 & 22,1 & 6,5 & 10,2 & 7,2 \\
\hline Separada/divorciada/viuda & 74,5 & 52,9 & 52,2 & 15,5 & 31,2 & 35,6 & 10,0 & 15,9 & 12,1 \\
\hline Soltera & 77,8 & 63,0 & 49,1 & 16,3 & 30,0 & 43,9 & 5,9 & 7,0 & 7,0 \\
\hline \multicolumn{10}{|l|}{ Nivel socioeconómicod } \\
\hline Bajo & 74,9 & 65,8 & 64,5 & 17,1 & 20,5 & 25,9 & 8,0 & 13,7 & 9,6 \\
\hline Medio & 76,9 & 69,1 & 65,3 & 16,3 & 22,5 & 27,5 & 6,8 & 8,4 & 7,2 \\
\hline Alto & 80,3 & 72,1 & 72,9 & 15,7 & 20,6 & 22,5 & 4,0 & 7,2 & 4,6 \\
\hline \multicolumn{10}{|l|}{ Situación laboral } \\
\hline Trabaja & 79,0 & 67,9 & 64,4 & 13,5 & 20,9 & 26,5 & 7,5 & 11,2 & 9,2 \\
\hline Sin trabajo remunerado & 75,7 & 68,7 & 68,3 & 18,0 & 21,3 & 25,1 & 6,3 & 10,0 & 6,7 \\
\hline \multicolumn{10}{|l|}{ Hijos actualmente vivos } \\
\hline 1 & 87,7 & 75,8 & 70,6 & 11,5 & 22,1 & 28,6 & 0,8 & 2,1 & 0,8 \\
\hline 2 & 81,0 & 73,7 & 69,8 & 16,6 & 23,0 & 27,2 & 2,5 & 3,2 & 3,0 \\
\hline 3 & 76,4 & 65,3 & 63,5 & 18,2 & 22,7 & 24,9 & 5,4 & 12,0 & 11,6 \\
\hline 4 & 69,8 & 61,4 & 63,6 & 21,4 & 21,3 & 19,4 & 8,7 & 17,3 & 17,1 \\
\hline$\geq 5$ & 68,4 & 57,6 & 55,3 & 17,1 & 15,4 & 19,3 & 14,6 & 27,0 & 25,4 \\
\hline
\end{tabular}

a ENDSR 1995: Encuesta Nacional de Salud Reproductiva, 1995 ( $n=2$ 859).

${ }^{b}$ ENDSSR 2004: Encuesta Nacional de Demografía y Salud Sexual y Reproductiva, 2004 ( $\left.n=2430\right)$.

c ENDSSR 2008: Encuesta Nacional de Demografía y Salud Sexual y Reproductiva, 2008 ( $n=1812$ ).

d Según los bienes duraderos que poseía y los servicios de las viviendas (24-26).

Las mujeres que vivían en áreas urbanas tuvieron mayor probabilidad de informar el último nacimiento como no deseado, que las de áreas rurales (OR = 2,837; IC95\%: 1,509 a 5,337).

La edad de las mujeres estuvo fuertemente asociada con el deseo de postergar el último nacimiento, especialmente en las adolescentes (de 15 a 19 años), que presentaron una mayor predisposición a considerar el último nacimiento como inoportuno que las del grupo de 30 a 44 años (OR = 8,219; IC95\%: 4,379 a 15,429). Así mismo, la posibilidad de considerar inoportuno el último nacimiento fue también mayor en las mujeres de 20 a 29 años que en las de 30 a 44 años (OR = 3,618; IC95\%: 2,376 a 5,509). En relación con el estado conyugal, el no estar casada o unida a una pareja estable estuvo fuertemente asociado con considerar el último nacimiento como no deseado o inoportuno, tanto si eran solteras (no deseado: $\mathrm{OR}=2,621$; IC95\%: 1,108 a 6,204; inoportuno: OR = 2,857; IC95\%: 1,751 a 4,661), o separadas, divorciadas o viudas (no deseado: $\mathrm{OR}=$ 2,753; IC95\%: 1,314 a 5,768; inoportuno: OR = 2,026; IC95\%: 1,219-3,367).

A medida que aumentaron los años de estudio disminuyó la posibilidad de que el último nacimiento se considerara inoportuno en lugar de deseado, en re- lación con las mujeres de mayor nivel educacional. Así, en las mujeres con menos de cinco años de educación esa posibilidad se redujo comparativamente en $58 \%$ y en las que terminaron entre 7 y 11 años de estudio disminuyó en 39\%.

Por cada nacimiento previo, la posibilidad de considerar inoportuno el último embarazo aumentó en $37 \%$ y en $93 \%$ la de informar que el último nacimiento no era deseado, en lugar de deseado.

\section{DISCUSIÓN}

Los niveles de fecundidad deseada y no deseada han sido objeto de va- 
CUADRO 5. Análisis de las intenciones reproductivas ante el último nacimiento ocurrido en los 36 meses anteriores a la encuesta, según las características socioeconómicas de las madres, Paraguay, Encuesta Nacional de Demografía y Salud Sexual y Reproductiva, 2008

\begin{tabular}{|c|c|c|c|c|c|c|}
\hline \multirow[b]{2}{*}{ Característica } & \multicolumn{3}{|c|}{$\begin{array}{l}\text { No deseado (no quería más) versus } \\
\text { deseado (quería entonces) } \\
\qquad(n=1226)\end{array}$} & \multicolumn{3}{|c|}{$\begin{array}{l}\text { Inoportuno (quería esperar) versus } \\
\text { deseado (quería entonces) } \\
(n=1486)\end{array}$} \\
\hline & $\mathrm{OR}^{\mathrm{a}}$ & IC95\% ${ }^{b}$ & $P^{c}$ & OR & IC95\% & $P$ \\
\hline Intersección & 0,004 & $0,001-0,013$ & $<0,001$ & 0,068 & $0,035-0,130$ & $<0,001$ \\
\hline \multicolumn{7}{|l|}{ Área de residencia } \\
\hline Urbana & 2,837 & $1,509-5,337$ & 0,001 & 1,014 & $0,720-1,428$ & 0,938 \\
\hline Rural (referencia) & 1,000 & & & 1,000 & & \\
\hline \multicolumn{7}{|l|}{ Edad actual (años) } \\
\hline $15-19$ & 1,924 & $0,624-5,936$ & 0,254 & 8,219 & $4,379-15,429$ & $<0,001$ \\
\hline $20-29$ & 1,236 & $0,714-2,140$ & 0,448 & 3,618 & $2,376-5,509$ & $<0,001$ \\
\hline 30-44 (referencia) & 1,000 & & & 1,000 & & . \\
\hline \multicolumn{7}{|l|}{ Años de estudio terminados } \\
\hline 0 a 5 & 0,774 & $0,321-1,869$ & 0,568 & 0,427 & $0,245-0,744$ & 0,003 \\
\hline 6 & 1,045 & $0,504-2,167$ & 0,905 & 0,641 & $0,402-1,021$ & 0,061 \\
\hline 7 a 11 & 0,834 & $0,407-1,710$ & 0,619 & 0,614 & $0,403-0,935$ & 0,023 \\
\hline$\geq 12$ (referencia) & 1,000 & & & 1,000 & & \\
\hline \multicolumn{7}{|l|}{ Estado conyugal } \\
\hline Soltera & 2,621 & $1,108-6,204$ & 0,028 & 2,857 & $1,751-4,661$ & $<0,001$ \\
\hline Separada/divorciada/viuda & 2,753 & $1,314-5,768$ & 0,007 & 2,026 & $1,219-3,367$ & 0,007 \\
\hline Casada/unida (referencia) & 1,000 & . & & 1,000 & & \\
\hline \multicolumn{7}{|l|}{ Nivel socioeconómico ${ }^{d}$} \\
\hline Bajo & 1,386 & $0,600-3,199$ & 0,444 & 1,222 & $0,774-1,929$ & 0,388 \\
\hline Medio & 1,702 & $0,861-3,364$ & 0,126 & 1,365 & $0,911-2,044$ & 0,131 \\
\hline Alto (referencia) & 1,000 & & & 1,000 & & \\
\hline \multicolumn{7}{|l|}{ Situación laboral } \\
\hline Trabaja & 1,036 & $0,611-1,756$ & 0,895 & 1,146 & $0,811-1,618$ & 0,439 \\
\hline $\begin{array}{l}\text { Sin trabajo remunerado } \\
\text { (referencia) }\end{array}$ & 1,000 & & & 1,000 & & \\
\hline \multicolumn{7}{|l|}{ Número de hijos actualmente } \\
\hline vivos & 1,931 & $1,648-2,263$ & $<0,001$ & 1,373 & $1,241-1,520$ & $<0,001$ \\
\hline
\end{tabular}

rios estudios en diferentes países. Esos estudios, como el que se presenta en este artículo, contribuyen a entender la conducta relacionada con la fecundidad y a conocer mejor sus efectos sobre la salud de la madre y el niño. También proveen información relevante para la salud pública, particularmente para los servicios de planificación familiar, y permiten conocer la necesidad insatisfecha de anticonceptivos (27). Entre las consecuencias más graves de un embarazo no deseado están los problemas derivados de los abortos inseguros (28).

En el presente estudio se constaron cambios en las condiciones sociales de las mujeres —en particular entre 1995 y 2008-, avances en su nivel educacional y laboral, un mayor uso de anticonceptivos y la disminución en la fecundidad. Un estudio reciente -basado en los datos de la Encuesta Nacional de Demografía y Salud Sexual y Reproductiva de 2004- concluyó que el amplio uso de anticonceptivos en Paraguay fue el factor más importante para la disminución de la tasa global de fecundidad. No obstante, se observó que, a pesar de ese aumento en el uso de anticonceptivos, también aumentaron los embarazos no deseados e inoportunos, lo que sugiere que en el futuro seguirá aumentando la demanda de anticonceptivos (29).

El análisis de regresión logística realizado aquí reveló que las adolescentes y las jóvenes -especialmente las solteras y las separadas, divorciadas o viudas - tendrían una probabilidad significativamente mayor de tener embarazos inoportunos que las mujeres de más edad. Asimismo, las mujeres que viven en áreas urbanas tendrían una mayor probabilidad de tener un embarazo no deseado que las que residen en áreas rurales. Esto concuerda con lo encontrado en un estudio realizado en la población adolescente con residencia urbana o periurbana en Ecuador (17). Otro estudio sugirió que en algunos contextos es más aceptado un embarazo no planeado en mujeres que viven en áreas rurales, ya que en las ciudades se espera que las mujeres jóvenes continúen estudiando hasta después de los 20 años (23).

$\mathrm{Al}$ igual que en otros estudios $(17,30)$, se encontró que aumentó la posibilidad de que el último hijo nacido se considerara inoportuno o no deseado.

Este estudio presenta limitaciones que se deben tener en cuenta al analizar los resultados. En primer lugar, la información sobre las intenciones reproductivas se basa en datos retrospectivos recolec- 
tados después de haber ocurrido el nacimiento; esto puede implicar un sesgo en la declaración de las mujeres que no siempre pueden recordar su estado de ánimo previo respecto a sus embarazos. En segundo lugar, las intenciones de las mujeres pueden cambiar en el transcurso del embarazo o después del nacimiento (17), ya sea por dificultades económicas o por la disolución de la unión con su pareja.

A pesar de las limitaciones mencionadas debidas a la naturaleza de los datos, se identificaron los factores asociados con una mayor posibilidad de que se produzcan nacimientos inoportunos $\mathrm{o}$ no deseados en la población estudiada y se comprobó que un mayor nivel de escolaridad reduce la posibilidad de que ocurran nacimientos inoportunos en adolescentes y jóvenes. Estos resultados muestran también que, a pesar del aupregnancy: worldwide levels, trends and outcomes. Stud Fam Plann. 2010;41:241-50.

2. Bankole A, Malarcher S. Removing barriers to adolescents' access to contraceptive information and services. Stud Fam Plann. 2010;41(2).

3. Paraguay. Código Penal, Ley 1160/97. Asunción: Ediciones Librería El Foro; 1997.

4. Paraguay. Constitución Nacional. Asunción: Ediciones Librería El Foro; 1992.

5. Paraguay, Ministerio de Salud Pública y Bienestar Social. Plan Nacional de Salud Sexual y Reproductiva 2009-2013. Asunción: Ministerio de Salud Pública y Bienestar Social; 2009.

6. Hakkert R. Fecundidad deseada y no deseada en América Latina. México, D.F.: Fondo de Población de las Naciones Unidas; 2001. (Working Papers Series CST/LAC No. 22).

7. Bongaarts J. Trend in unwanted childbearing in the developing world. Stud Fam Plann. 1997;28(4):267-76.

8. Bongaarts J. The measurement of wanted fertility. Popul Dev Rev. 1990;16(3):487-506.

9. Bongaarts J, Lightbourne R. Fecundidad deseada en América Latina: tendencias y diferenciales en siete países. Notas Población. 1992;20(55):79-102.

10. Westoff CF. Unwanted fertility in six developing countries. Perspect Int Planif Fam. 1981;7(2):43-54.

11. Casterline JB, El Zeini LO. Estimation of unwanted fertility. Demography. 2007;88(4): 729-45.

12. Adetunji J. Nacimientos inoportunos y no deseados en el curso de la transición de la fecundidad. En: Naciones Unidas, ed. Perspectivas de descenso de la fecundidad en los países con fecundidad elevada. Nueva York: NU; 2002. Pp. 111-32. (Boletín de Población de las Naciones Unidas No. 46/47.)

13. Campbell A, Mosher W. A history of the measurement of unintended pregnan- mento en el nivel de escolaridad de las mujeres, la mayor incorporación al trabajo remunerado, el mayor uso de métodos anticonceptivos y la disminución de la fecundidad, todavía existen grupos de mujeres que no pueden satisfacer sus ideales reproductivos y están expuestas a embarazos no deseados o inoportunos.

Los programas de planificación familiar en Paraguay deben priorizar la atención de las adolescentes y las jóvenes, así como de las mujeres sexualmente activas que no se encuentran en una relación estable de pareja, tomando en cuenta sus características específicas y necesidades. Se recomienda evaluar el efecto de la inestabilidad de las uniones de pareja en las preferencias reproductivas en los países de América Latina y emprender estudios cualitativos que profundicen los aspectos motivacionales de las mujeres en relación con sus intenciones reproductivas.

\section{REFERENCIAS}

cies and births. Matern Child Health J. 2000;4(3):163-9.

14. D'Angelo DV, Colley Gilbert B, Rochat RW, Santelli, JS, Herold JM. Differences between mistimed and unwanted pregnancies among women who have live births. Perspect Sex Reprod Health. 2004;36( 5):192-7.

15. Latin American Population Association. Demographic transformations and inequalities in Latin America: historical trends and recent patterns. 1. ${ }^{\mathrm{a}}$ ed. Rio de Janeiro: ALAP; 2009. (Research Series No. 8.)

16. Blanc AK. Unwanted fertility in Latin America and the Caribbean. Int Fam Plan Perspect. 1982;8(4):156-62.

17. Eggleston E. Causas determinantes de embarazos no planeados en el Ecuador. Int Fam Plan Perspect. 1999:2-8.

18. Paraguay, Centro Paraguayo de Estudios de Población. Encuesta Nacional de Demografía y Salud Reproductiva, 1995. Asunción: CEPEP, USAID, CDC; 1997.

19. Paraguay, Centro Paraguayo de Estudios de Población. Encuesta Nacional de Demografía y Salud Sexual y Reproductiva, 2004. Asunción: CEPEP, USAID, CDC, UNFPA, IPPF; 2005.

20. Paraguay, Centro Paraguayo de Estudios de Población. Encuesta Nacional de Demografía y Salud Sexual y Reproductiva, 2008. Asunción: CEPEP, USAID, CDC, UNICEF, IPPF; 2009.

21. Comisión Económica para América Latina y el Caribe. Sistema de indicadores sociodemográficos de poblaciones y pueblos indígenas: tasa global de fecundidad. Santiago: CELADE; sin fecha. Disponible en: http:// celade.cepal.org/redatam/PRYESP/SISPPI/ Webhelp/fecundidad.htm Acceso el 18 mayo de 2012.

22. Franco Suárez MC, González Galván DE, Fernández Suárez JC. Caracterización de la
Agradecimientos. El presente trabajo forma parte de una investigación realizada por el Centro Paraguayo de Estudios de Población en el marco de un proyecto de cooperación con el departamento de Salud Reproductiva e Investigación de la Organización Mundial de la Salud. Se agradece a Alejandra Pantelides y al equipo del Centro de Estudios de Población de Argentina por sus consejos y orientaciones; a Julio Galeano, Édgar Tullo, Marco Castillo, Ruth Navarro y Alejandra Martínez por la ayuda recibida en el procesamiento e interpretación de los datos y la revisión bibliográfica; y a Ishida Kanako, del Centro para el Control y Prevención de Enfermedades (CDC) de Estados Unidos, por su valiosos comentarios.

\section{Conflictos de interés. Ninguno.}

población femenina con ideales reproductivos por encima del reemplazo. Rev Cubana Salud Publica. 2006;32(1):1-13.

23. Núñez Urquiza R, Hernández Prado B, García Barrios C, González D, Walker D. Embarazos no deseados en adolescentes y utilización de métodos anticonceptivos posparto. Salud Publica Mex. 2003;45(1):92-102.

24. Filmer P. Estimating wealth effects without income or expenditure data or tears. Educational enrollment in India. Washington, D.C.: World Bank; 1994.

25. Filmer P. The effect of household wealth on educational attainment: evidence from 35 countries. Popul Dev Rev. 1999;25(1):85-120.

26. Stupp P, Daniels D, Ruiz A. Reproductive, maternal, and child health in Central America: health equity trends. Atlanta, GA: Centers for Disease Control and Prevention; 2007.

27. Santelli J, Rochat R, Hatfield-Timajchy K, Colley Gilbet B, Curtis K, Cabral R, et al. The measurement and meaning of unintended pregnancy. Perspect Sex Reprod Health. 2003;35( 2):94-101.

28. Langer A. El embarazo no deseado: impacto sobre la salud y la sociedad en América Latina y el Caribe. Rev Panam Salud Publica. 2002;11(3):192-204.

29. Kanako I, Stupp P, Melian M. Fertility decline in Paraguay. Stud Fam Plann. 2009;40(3)3.

30. Kamal M, Islam A. Prevalence and socioeconomic correlates of unintended pregnancy among women in rural Bangladesh. Salud Publica Mex. 2011;53(2):108-115.

Manuscrito recibido el 9 de febrero de 2012. Aceptado para publicación, tras revisión, el 12 de octubre de 2012. 
ABSTRACT Objective. To describe the reproductive intentions of Paraguayan women over the period from 1995 to 2008 and determine the characteristics associated with unwanted and mistimed pregnancies.

\section{Reproductive intentions and factors related to unplanned births, Paraguay, 1995-2008}

Methods. An exploratory, descriptive study was conducted using secondary data from national demographic and reproductive health surveys from 1995, 2004, and 2008. The study examined the socio-demographic characteristics of the women, the use of contraceptive methods, and fertility, as well as the proportion of wanted, mistimed, and unwanted births. Using two multifactorial logistic regression models, the characteristics associated with unwanted or mistimed pregnancies were determined. Results. In 2008, one-third (33.3\%) of the births were unplanned (25.6\% mistimed and $7.7 \%$ unwanted). Age was directly and significantly associated with the woman having wanted to postpone her most recent birth; level of schooling was inversely related to the possibility of having mistimed births; and living in urban areas was associated with unwanted births. Not being married or in a stable relationship was associated with regarding the most recent birth as unwanted or mistimed, not only among single women, but also among those separated, divorced, and widowed.

Conclusions. Despite improvements in women's levels of schooling, their increasing access to paid employment, the growing use of contraceptive methods, and reductions in fertility, there are still groups of women who are unable to fulfill their reproductive intentions and are at risk of having unwanted or mistimed pregnancies.

Key words Fertility; family planning (public health); pregnancy, unplanned; pregnancy, unwanted; Paraguay. 